\title{
Modelling and Simulation on Evolution Effect of Knowledge Sharing on Industry Cluster Based on Social Network
}

\author{
Houxing Tang ${ }^{*}$
}

School of Business Administration, Nanchang Institute of Technology, Nanchang, Jiangxi, 330099, China

\begin{abstract}
In order to explain the argument about effect of knowledge sharing on industry cluster, the knowledge accumulation process was modeled based on innovation ability and exchange frequency of knowledge. And then, three curves were simulated, which represent the average knowledge stock of cluster, average deviation of knowledge stock between enterprises, and knowledge accumulation process of each enterprise respectively. The simulation results show that firstly, the innovation ability is the inner driving force to increase knowledge stock of enterprise. The knowledge stock of cluster will increase and the cluster will become much more divergence with the increasing of innovation ability. Secondly, the knowledge exchange frequency is the external driving force to increase the knowledge growth of enterprise. The knowledge stock of cluster will increase and the cluster will also become much more divergence with the increasing of exchange frequency. Thirdly, knowledge sharing will lead the cluster into absolute divergence with relative convergence. These variables will affect the degree of convergence and divergence, but don't change the final evolution trend. These results are helpful to explain the argument about effects of knowledge sharing and explore the black box of innovation process in cluster.
\end{abstract}

Keywords: Evolution effect, industry cluster, knowledge sharing, modelling and simulation, social network.

\section{INTRODUCTION}

With the advent of knowledge economy, the knowledge is increasingly seen as an important strategic resource to maintain a competitive advantage. The enterprise' demands for new knowledge are increasing. In particular, comparing with the traditional industrial cluster, the enterprises in hightech industrial clusters tend to be treated as knowledge-based enterprises. Therefore, it is quietly urgent for these enterprises to demand more knowledge and innovation. However, the innovation is the collision and integration of different thoughts fragments from different individuals. So, it is difficult for an enterprise to implement the innovation based on its resource. No doubt, the innovation is a complex and interactive social learning process.

In the "national innovation system" report of OECD (1997), it pointed out that the essence of cluster innovation system is the flowing and transferring of knowledge among different agents. If and only if the knowledge resources are captured and applied in most enterprises, this knowledge would play their importance and get economic value. Deng, Yi \& Cai thought that, from the process of knowledge flowing, the knowledge evolution is a key link of innovation. It doesn't format the competitive advantage and performance directly, but format the organizational creativity and then obtains the competitive advantages [1]. In other words, it is the knowledge flowing and sharing that leads to the evolution of cluster innovation. So, how to acquire enough new knowledge from inside and outside of high-tech cluster by knowledge sharing is becoming a key factor to promote the innovation and development of cluster and enterprises themselves.

Furthermore, some scholars such as Li pointed out that the free-rider may make knowledge-follower catch up with the knowledge-leader. This would make these enterprises be convergent and lead to malignant competition, which is not conducive to continuous innovation and development of cluster [2]. However, some scholars such as Eechhout \& Jovanovic argued that, because there were many differences such as absorption ability, cognitive process and cost of knowledge sharing, the knowledge-follower would search for appropriate knowledge rather than copy the cutting-edge knowledge of the knowledge-leader. Particularly, the knowledge-leader doesn't need to acquire knowledge from knowledge-follower. So, this leads to further imbalance of knowledge distribution within group. That is to say, knowledge sharing makes the enterprises in cluster be divergent [3].

Well, how to deal with such conflict about the effect of knowledge sharing on cluster? In these literatures, we have found the core problem is the different assumption of process of knowledge sharing. In other words, it is very important to exploring the interactive process of knowledge sharing and analyzes its effect on cluster. However, due to the characteristics of knowledge itself and complexity of social learning and interaction, the process of knowledge sharing within industrial cluster is still full of myth. Especially, the industry cluster is a typical network organization. Zhao \& Luo thought that the network structure of high-tech industry 
cluster makes enterprises break down many constraints with the help of network connections, and then obtain a lot of key elements such as knowledge, resources and personnel from the network environment [4]. In addition to, Chen, $\mathrm{Hu} \&$ Yang pointed out that the network of industry cluster is a running platform for intellectual activity within the cluster. Inevitably, the network structure will affect the performance of knowledge activities, and thereby affect the knowledge evolution in cluster [5]. For examples, Huang, Zhuang \& Yao found that the innovation collaboration network with scalefree can most greatly improve the depth and velocity of knowledge diffusion, the growth rate of overall knowledge stock, and has the highest efficiency for the allocation of knowledge resources. These agents who locate in spatial agglomeration tend to have similar level of knowledge stock in innovation collaboration network [6].

Cowan, Jonard \& Ożman, Cowan \& Jonard, Baum, Cowan \& Jonard put much focuses on the interaction regulars and different network structures, and examined how these factors influence on knowledge diffusion speed and knowledge accumulation efficiency. They explored which structure is the optimal efficiency for knowledge diffusion. And then, they found that the small world network is the most favorable for knowledge diffusion [7-12]. Kim, Lee, Choe \& Seo analyze how the changes of network structure affect the firm's performance and especially focus on the node's position during the cluster evolution process. The empirical results show that social networking technology increases the informal relationships of the cluster network structure, and eventually heighten centrality as well as potentially affect the growth of the high-tech industry cluster [13].

However, Morone \& Taylor argued that the small world network which is optimal in theory is very difficult to construct in real world and its effectiveness can not be proved [14]. Chen, Hu \& Yang also pointed out that, in reality, the network structures of different industry clusters are different. So, those classical network models in complex network theory such as small-world network, free-scale network don't accurately describe the networks structures of industry clusters in reality [15].

Generally, there are many arguments about the network structure, process of knowledge interaction and effect of knowledge sharing, which need to be studied deeply. Therefore, in this paper, some assumptions on formation of social network structure, conditions of knowledge interaction among enterprises with high-tech industry cluster are proposed in section 1. In section 2, an interaction and accumulation model of knowledge sharing is constructed based on many factors such as absorption efficiency of knowledge, frequency of interaction and innovation capacity. And then, the evolution of cluster knowledge stock is described by average knowledge stock (AKS) and deviation of knowledge stock (DKS). In section 3, with the help of computer simulation, the evolution of knowledge sharing among enterprises is plotted. And then, the role of different factors on knowledge evolution is analyzed. The debate on convergent and divergent, or effect of knowledge sharing on industry cluster, is explained clearly. In section 4, conclusions and many policies promoting sharing knowledge are proposed.

\section{MODELLING ON EVOLUTION OF KNOWLEDGE SHARING}

\subsection{Basic Assumptions}

Suppose that the number of enterprises in high-tech industry cluster is $N$ and $N=\{1,2, \ldots, \mathrm{n}\}$. Each enterprise is just a node $v_{\mathrm{i}}(\mathrm{i} \in \mathrm{N})$. The $k_{i}^{t}$ represents the knowledge stock of enterprise $k$ at time $t$. If there exists a relationship between enterprise $i$ and $j$, it will format an edge, that is $e_{\mathrm{ij}}(i, j \in \mathrm{N}, \mathrm{i} \neq \mathrm{j})$. So, it formats an indirection graph based on social relationship among enterprises. Let $d(i, j)$ stand for the shortest path length between node $i$ and $j$. If there is direct relationship between the two nodes, then $d(i, j)=1$, or $d(i, j)=0$. That is to say, the enterprises $i$ and $j$ can carry out knowledge sharing when $d(i, j)=1$.

Connell, Kriz \&Thorpe (2014) pointed out that the ability of an enterprise that can effectively connect with other organizations is treated as the core element of contemporary innovation management strategy. Their case studies showed that the communication among enterprises in cluster can greatly speed up the exchange and sharing of knowledge. The communication is promoted by formal and informal means based on strategic and tacit aspects. No doubt, the quality and frequency of the communication among enterprises becomes a key indicator to measure the cluster performance [16]. Therefore, in this paper, the quality and frequency of knowledge sharing is incorporated in the evolution model.

\subsection{The Relationship between Knowledge Absorption Efficiency and Relative Knowledge Gap}

Both the theory and the practice show that efficient knowledge-added is not gained or the absorption efficiency of knowledge is not high through knowledge exchange, if the difference of knowledge stock between two enterprises is either too small or too large.

How to explain it? As we all know, if the difference of knowledge stock is too small and lack of diversity, the two enterprises are thought to have similar innovation capacity. Therefore, much more new knowledge can not be provided through knowledge exchange. Conversely, if the difference of knowledge stock is too large, the knowledge-leader enterprise will not gain much new knowledge because it has gotten hold of what the knowledge-follower knows well. And for the knowledge-follower enterprise, it has no ability to assimilate and absorb the knowledge from exchange because of insufficient knowledge base. So, the knowledge-follower does not also gain much new knowledge. In summaries, the appropriate knowledge gap is helpful to increase the absorption efficiency of knowledge sharing. Therefore, we think that there is an inverted $U$-shaped relationship between knowledge absorption efficiency (i.e. KAE) and relative knowledge gap (i.e. RKG).

It is somewhat similar with the density function of standard normal distribution function. So, we assume that

$\rho=\frac{1}{\sqrt{2 \pi} \sigma} e^{-\frac{(\Delta \tilde{k}-\mu)^{2}}{2 \sigma^{2}}}$

In formula (1), the $\rho$ stands for the KAE and the $\Delta \tilde{k}$ stands for the RKG. The $\mu$ and $\sigma$ are the expected value and 
standard deviation values of the normal distribution function respectively. They are treated as moderating variables here. Firstly, the parameter $\mu$ controls the moving of curve to left or right. That is to say, it controls the change of the optimal RKG. Especially, the optimal RKG is different with the development of the enterprises and overall hightech industry cluster. So, the parameter $\mu$ can be used to measure the heterogeneity and complementarily of knowledge innovation ability of different enterprises in high-tech industry cluster. Also, it reflects the characteristics of cluster. If it can obtain better effect of knowledge sharing based on larger $\Delta \tilde{k}$ with the increasing of $\mu$, then this result indicates that the cluster has lower specialization and diversified knowledge distribution is much helpful for innovation. On the contrary, if it can obtain better effect of knowledge sharing based on smaller $\Delta \tilde{k}$ with the decreasing of $\mu$, this result indicates that the cluster has higher specialization and specialized knowledge distribution is much helpful for innovation.

Secondly, the parameter $\sigma$ controls the appearance (i.e. fat or thin) of the graphics. For example, the graphics will become more flattened with the increasing of $\sigma$. From the formula (1), we can calculate the maximum value of the $\rho$ is $1 / \sqrt{2 \pi} \sigma$. So, the $\rho$ will exceed to 1 when $\sigma<1 / \sqrt{2 \pi} \sigma$, which indicates that the enterprise has much higher knowledge absorption capacity. Because it not only can fully absorb the knowledge from sharing, but also can make further innovation based on these knowledge. On the contrary, if $\sigma \geq 1 / \sqrt{2 \pi} \sigma$, then $\rho \leq 1$, which indicates that the enterprise only absorb a part of shared knowledge and has much lower innovation capacity. Therefore, the parameter $\sigma$ can be used to identify and control the change of innovation capability of enterprises, as well as, it also represents the quality of absorbing shared knowledge.

\subsection{The Relationship between Knowledge-Added and Knowledge Gap}

It is difficult to quantify the new knowledge-added what the enterprises actually obtain in one interaction. Perhaps, the enterprises with higher knowledge stock acquire more new knowledge. Perhaps, the enterprises with less knowledge stock can obtain much more knowledge-added. So, in order to simplify the complexity of the model, we assume that the two interactive enterprises will get the same knowledge-added in each interaction. The quantity of knowledgeadded has a liner relationship with the absolute value of the knowledge gap between the two enterprises. So, we get

$\Delta k_{i j}^{t}=\lambda\left|k_{i}^{t-1}-k_{j}^{t-1}\right|$

Where, the $\Delta k_{i j}^{t}$ stands for the knowledge-added for one interaction between enterprises $i$ and $j$ at time $t$; the parameter $\lambda$ stands for the utility efficiency of knowledge stock. The $\left|k_{i}^{t-1}-k_{j}^{t-1}\right|$ is the absolute value of knowledge gap between enterprise $i$ and $j$ at time $t-1$.

\subsection{The Relationship between Knowledge Stock and Interaction Frequency}

The absorption efficiency of shared knowledge is affected by innovation capacity of enterprise and the quantity of shared knowledge is affected by the number of interaction between enterprises. So, we can get the quantity of knowledge stock for enterprise $i$ at time $t$, that is

$k_{i}^{t}=k_{i}^{t-1}+\sum_{j \in \Phi(i)} q_{i j}^{t} \cdot \Delta k_{i j}^{t-1}$

Where, the $q_{i j}^{t}$ stands for the frequency of interaction between enterprises $i$ and $j$ at time $t$. The $\Phi(i)$ stands for an assemble which concludes all neighbors of enterprise $i$.

\subsection{The General Evolution Model of Knowledge Sharing among Enterprises}

Now, based on above analysis, we get the function for evolution of knowledge sharing among enterprises within high-tech industry cluster as follow

$$
\begin{aligned}
& k_{i}^{t}=k_{i}^{t-1}+\sum_{j \in \Phi(i)} \frac{1}{\sqrt{2 \pi} \sigma} e^{-\frac{(\Delta \tilde{k}-\mu)^{2}}{2 \sigma^{2}}} \cdot \lambda\left|k_{i}^{t-1}-k_{j}^{t-1}\right| \cdot q_{i}^{t} \\
& i \in N, t \in T
\end{aligned}
$$

In order to describe the effect of knowledge sharing on cluster, two indicators are constructed namely average knowledge stock (AKS) and deviation of knowledge stock (DKS). They are shown as follow

$$
\begin{aligned}
A K S^{t} & =\frac{1}{n} \sum_{i=1}^{n} k_{i}^{t} \\
D K S^{t} & =\frac{\sum_{i=1}^{n}\left|k_{i}^{t}-A K S^{t}\right|}{\sum_{i=1}^{n} k_{i}^{t}}
\end{aligned}
$$

\section{SIMULATING AND ANALYZING BASED ON MATLAB}

\subsection{Setting of Basic Parameters}

Assume that the number of enterprises in the cluster is 50 , that is $n=50$. And the time series of evolution is 100 , that is $t=100$. This paper argues that the enterprises in high-tech industry clusters are diversified, that are heterogeneous on innovation capacity. And it can be reflected by differences of initial knowledge stock $\left(k_{i}^{0}\right)$ among enterprises. So, a uniform random function in the interval $(0,1)$ is used to generate the initial knowledge stock. In order to avoid the errors from arbitrary assignment and simplify the complexity of model, the coefficient of absorption efficiency of knowledge sharing is set a constant variable, that is $\lambda=0.01$. Generally speaking, the specialization is much higher in high-tech industry cluster than that of ordinary cluster. That is to say, having lower RKG may be much conducive to communication among enterprises. Therefore, the parameter $\mu$ is set to 0.3 , that is $\mu=0.3$. Similarly, the parameters $\sigma$ and $q$ are set to 0.5 and 2 respectively, that are $\sigma=0.5$ and $q=2$. In addition to, because the numerical value of DKS is too small, it is difficult to observation and analysis. So, the value is amplified with a ratio 40 .

In follow sub-sections, we mainly examine the influence of the parameters $\sigma$ and $q$ on the accumulation of knowledge stock of each enterprise. Furthermore, overall effects of 
knowledge sharing on evolution of high-tech industry cluster can be explored and explained.

\subsection{Simulation and Analysis}

The simulation is implemented by Matlab R2010 and these results are shown in Figs. (1 and 2). In these figures, the solid lines stand for AKS and the thick-dotted lines stand for DKS. The thin-dotted lines stand for the knowledge stock of each enterprise. Concrete analysis is as follows:

Firstly, from these figures, we can find that all the AKS curves are always monotonically increasing. This indicates that knowledge sharing will increase the knowledge stock for each enterprise and the whole cluster, but different variable has different effect on the strength of knowledge growth. In addition to, different variable has different effect on the morphology of DKS curve. As shown in figures, some are asymmetrical U-shaped, some are flatten Sshaped and some are $\mathrm{W}$-shaped. However, the overall trend of evolution is declined at first and uplifted after that. Especially, for the evolution curves of individual enterprise, many curves cross each other at some time. So, these enterprise present relatively concentrated after a perplex process which experience following, catching and surpassing. All these indicate that the knowledge sharing finally leads the enterprises into absolute divergence, although there are many relative convergences in the process of knowledge sharing.

Secondly, in Fig. (1), one side, the maximum value of AKS curve is gradually decreasing with the increasing of $\sigma$. Don't forget that the increasing of $\sigma$ means the decreasing of innovation capacity. Obviously, the accumulation of knowledge-added will become lower with the weakening of innovation capacity of enterprise. Another side, the maximum value of DKS curve also gradually decreases with the increasing of $\sigma$ and the descending part of this curve is gradually becoming longer. That is to say, there are many convergences in the evolution process of knowledge sharing for a part of enterprises. How to explain it? We think that this can be explained through the evolution curves of single enterprise. The differentiation among individual evolution curves is the most serious when $\sigma$ equals to 0.1(i.e. the enterprise has much stronger innovation capability). Sometimes, these evolution curves get together and sometimes get scattered, which shows convergence and divergence repeatedly. This is why DKS curve appears W-shaped. However, such chaotic differentiation phenomenon gradually weakens with the increasing of $\sigma$, but local polymerization becomes obvious, which ultimately reduce the value of DKS. In general, higher innovation capability will urge the enterprises in cluster to be divergent. Furthermore, it indicates that higher innovation capability will urge the enterprises in cluster to be diversified. And this is the precise reason that the high-tech cluster can maintain a higher dynamic.

Thirdly, from Fig. (2), the value and slope of AKS curve increase with the increase of interaction frequency $q$. This shows that active knowledge communication among enterprises can significantly increase the accumulation of knowledge stock. Also, it indicates that it can promote the growth of new knowledge to construct a good atmosphere of knowledge communication in the high-tech industrial clusters.
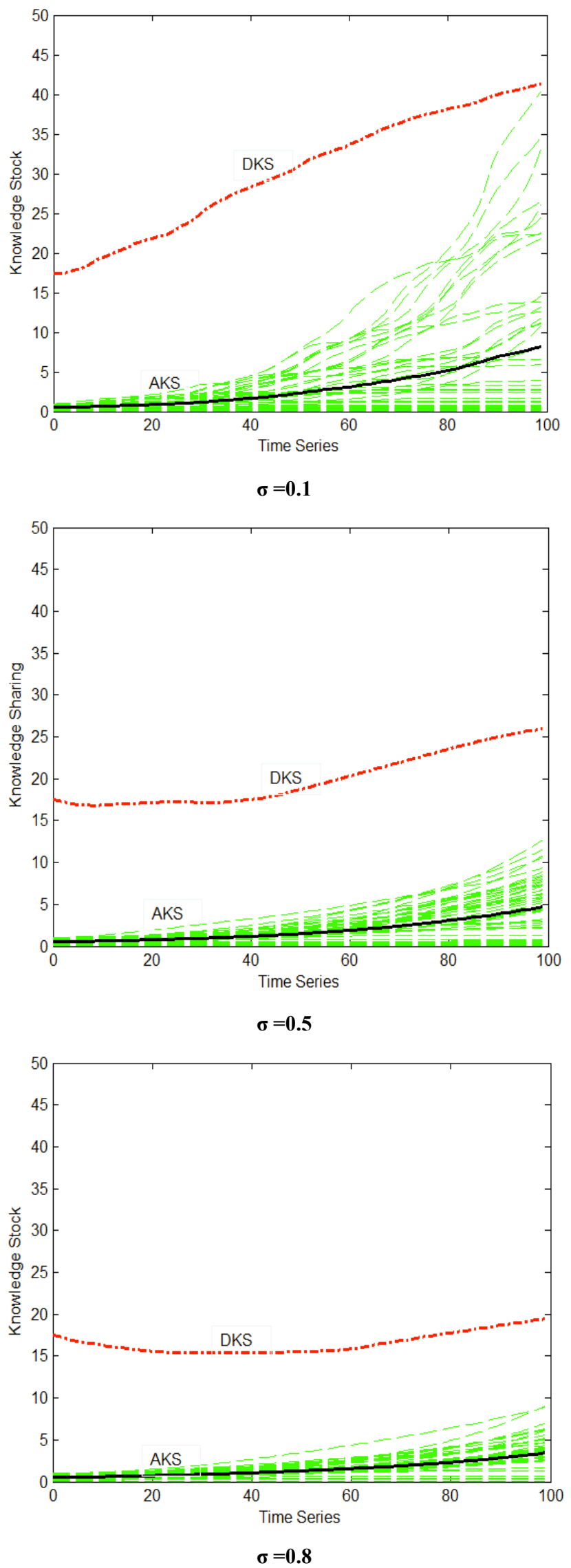

Fig. (1). The effect of $\sigma$ on evolution of knowledge sharing. 

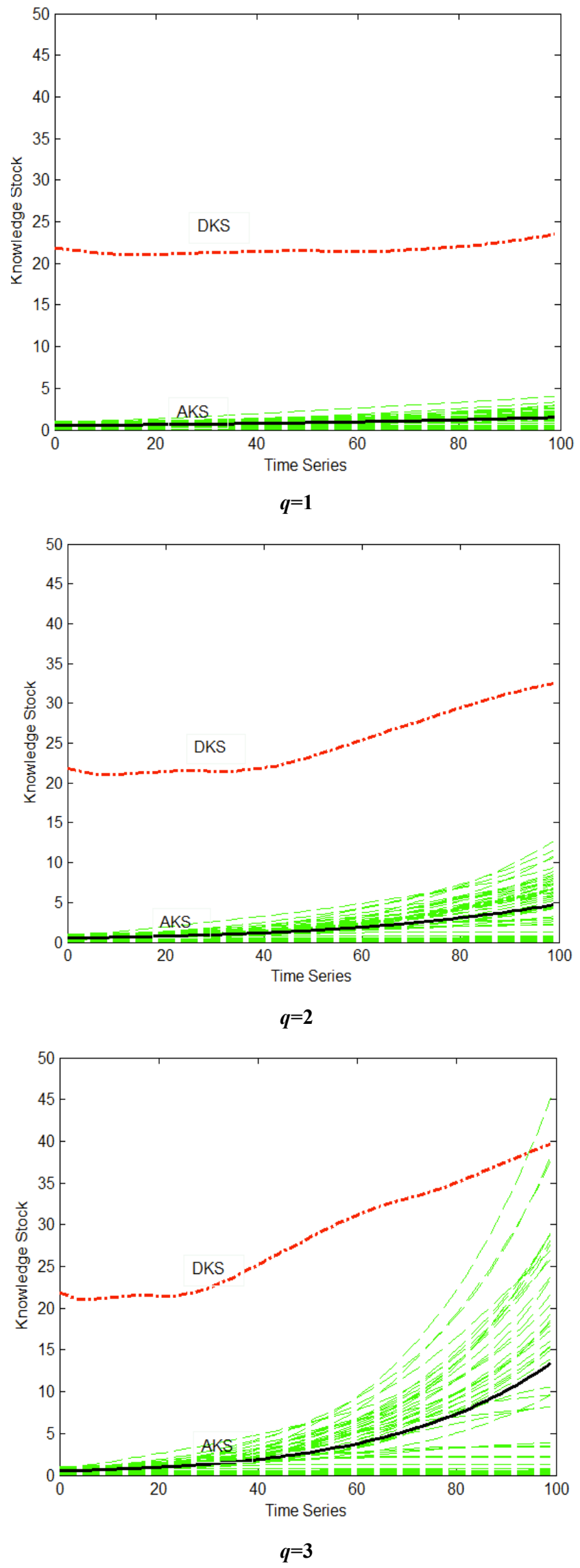

Fig. (2). The effect of $q$ on evolution of knowledge sharing.
This is one of the most important factors to maintain the dynamic and success of the Silicon Valley. In addition, the knowledge gap among enterprises is gradually increasing with the increasing of $q$. Particularly, the increase has an accelerating pace. All these show that those enterprises which have more social relationship (i.e. higher network density) will obtain much more knowledge through more interactions. No doubt, this makes the knowledge gap between higher density enterprise and lower density enterprise be widened. A few enterprises have a leap-forward development and their knowledge stock will outdistance the average level of overall cluster. Furthermore, this increases the knowledge stock of other connected enterprises. Finally, it makes these enterprises become different groups and the whole cluster becomes more and more divergent. So, these results also can certificate the role and effect of core enterprise on knowledge sharing in cluster.

\section{CONCLUSION AND DISCUSSION}

The knowledge resources have become the key source to maintain innovation and competitive advantage for the hightech industry cluster. So, how to effectively acquire knowledge has become an important topic for enterprises in hightech cluster. However, the knowledge sharing process in cluster is still not very clear. And effects of knowledge sharing on high-tech industry cluster remain a debate. Therefore, in this paper, a evolution process of knowledge sharing is modeled. And many conclusions are as follows:

Firstly, it will form many different groups with different knowledge levels when the enterprises' innovation abilities are weak. Each group is convergent and the gaps between groups are also lower. But, with the increasing of enterprises' innovation ability, the differences between enterprises become more and more. The groups become more dispersed. Consequently, the gaps between groups become also widen. All these indicate that the divergence will become higher with the increasing of enterprises' innovation ability. In some sense, the enterprises' innovation ability is an important factor to measure the dynamic of cluster. Maybe, the development of Silicon Valley fully proved this viewpoint.

Secondly, the higher the frequency of knowledge interaction between enterprises is, the more the accumulation of knowledge stock is. Some enterprises increase the knowledge stock through interactions and realize the overtaking. But, some enterprises just acquire a little knowledge-added because they are lack of partners or their partners have no sufficient knowledge. One side, it shows that frequent communication is beneficial to the growth of knowledge in cluster. So, we should form a good atmosphere of sharing and actively build all kind of service platforms to support and encourage the formal and informal communication. Be like this only, the Coffee Hall Effect may be implemented. Another side, it also shows that the core enterprises have much more social capital and therefore have more opportunity to communicate with other enterprises. No doubt, they can acquire more knowledge-added. In turn, we hope that the core enterprises can play a leading role on increasing the knowledge accumulation for overall cluster through the conductive effect of social network. 
We hope to be able to give full play to the leading role of core enterprise, through the conduction effect of social networks, to promote all the enterprise knowledge accumulation, and to enhance the cluster's innovation ability.

Surely, there are many limitations. For example, the accumulation function may be constructed arbitrarily. So, in the future work, different function should be tested and the best one is chosen.

\section{CONFLICT OF INTEREST}

The author confirms that this article content has no conflict of interest.

\section{ACKNOWLEDGEMENTS}

We acknowledge the financial support by National Natural Science Foundation of China (Grand No: 71261019), also the Youth Fund Project of College Humanities and Social Sciences of Ministry of Education of China (Grand No: 11YJC630193), respectively.

\section{REFERENCES}

[1] W. Deng, M. Yi, and G. Cai, "Study on the model of knowledge evolvement of industry clusters based on knowledge network", Journal of Intelligence, vol. 28, no.6, pp. 106-109, 2009.

[2] X. Li, "A review on effect and potential risks of knowledge spillovers for high-tech industry cluster", Academic Exchange, vol. 8, pp.100-103, 2011.

[3] E. Jan, and J. Boyan, "Knowledge spillovers and inequality", The American Economic Review, vol. 92, no.5, pp.1290-1307, 2002.

[4] P. Zhao, and F. Luo, "Research on knowledge innovation path of high-tech industrial cluster based on network organization structure", Reform \& Strategy, vol. 29, no. 12, pp. 117-119, 2013.
[5] J. Chen, H. Hu, and Y. Yang, "Research on know ledge evolution in industrial cluster based on network", Studies on Science of Science, vol. 29, no. 1, pp. 91-96, 2011.

[6] W. Huang, X. Zhuang, and S. Yao, "Study on knowledge diffusion of industry clusters based on the innovation cooperation network", Journal of Management Science, vol. 25, no. 2, pp.13-23, 2012.

[7] R. Cowan, N. Jonard, and M. Ożman, "Knowledge dynamics in a network industry", Technological Forecasting \& Social Change, vol. 71, pp.469-484, 2004.

[8] R. Cowan, and N. Jonard, "Network structure and the diffusion of knowledge," Journal of Economics Dynamics and Control, vol. 28, no. 8, pp.1557-1575, 2004.

[9] R. Cowan, and N. Jonard, "Structural holes, innovation and the distribution of ideas", Journal of Economics Interaction and Coordination, vol. 2, no. 2, pp.73-110, 2007.

[10] R. Cowan, and N. Jonard, "Network architecture, barter exchange and the diffusion of ideas", International Journal of Agricultural Resources Governance and Ecology, vol. 6, no. 2, pp.165-178, 2007.

[11] J.A.C. Baum, R. Cowan, and N. Jonard, "Network-independent partner selection and the evolution of innovation networks", Management Science, vol. 56, no. 11, pp. 2094-2110, 2010.

[12] J.A.C. Baum, R. Cowan, and N. Jonard, "Does evidence of network effects on firm performance in pooled cross-section support prescriptions for network strategy", Strategic Management Journal, vol. 35, pp. 652- 667, 2014.

[13] H.D. Kim, D.H. Lee, H. Choe, and W. Seo, "The evolution of cluster network structure and firm growth: a study of industrial software clusters", Scientometrics, vol. 99, pp. 77-95, 2014.

[14] P. Morone, and R. Taylor, "Knowledge diffusion dynamics and network properties of face-to-face interactions", Journal of Evolutionary Economics, vol. 14, no. 3, pp. 327-351, 2004.

[15] J. Chen, H. Hu, and Y. Yang, "Research on knowledge evolution in industrial cluster based on network", Studies on Science of Science, vol. 29, no. 1, pp. 91-96, 2011.

[16] J. Connell, A. Kriz, and M. Thorpe, "Industry clusters: an antidote for knowledge sharing and collaborative innovation?", Journal of Knowledge Management, vol. 18, no. 1, pp. 137-151, 2014.

(C) Houxing Tang; Licensee Bentham Open.

This is an open access article licensed under the terms of the (https://creativecommons.org/licenses/by/4.0/legalcode), which permits unrestricted, noncommercial use, distribution and reproduction in any medium, provided the work is properly cited. 